\title{
MultiPlane: A New Framework for Drawing Graphs in Three Dimensions*
}

\author{
Seok-Hee Hong \\ School of Information Technologies, University of Sydney, Australia \\ NICTA (National ICT Australia) \\ shhong@it.usyd.edu.au
}

This poster presents a new framework for drawing graphs in three dimensions, which can be used effectively to visualise large and complex real world networks.

The new framework uses a divide and conquer approach. More specifically, the framework divides a graph into a set of smaller subgraphs, and then draws each subgraph in a 2D plane using well-known 2D drawing algorithms. Finally, a 3D drawing of the whole graph is constructed by combining each drawing in a plane, satisfying defined criteria.

The framework is very flexible. Algorithms that follow this framework vary in computational complexity, depending on the type of graph and the optimisation criteria that are used. Specific instantiations of the framework require solutions to optimisation problems. A simple example of the framework is illustrated in Figure 1

Our framework generalises some existing methods. For example, PolyPlane methods draw trees in 3D [6]. Another method is to use two and a half dimensional methods to visualise related networks in parallel planes [3, 4, 9].

Further, the design principle behind the framework also confirms Ware's guideline, a $2 \frac{1}{2}$ design attitude that uses $3 \mathrm{D}$ depth selectively and pays special attention to 2D layout may provide the best match with the limited 3D capabilities of the human visual system [9].

As examined with the PolyPlane methods, the resulting drawing can reduce visual complexity and occlusion, and ease navigation. While rotating the drawing, some of the planes can be displayed as lines; this both reduce visual complexity and occlusion and allow the user concentrate on their plane of interest.

Preliminary results suggest that the framework can be useful for visual analysis and insight into large and complex networks such as hierarchical graphs and clustered graphs arising in social networks and biological networks domains. For details, see [2] for scale-free networks, [7,8 for directed graphs and [5] for clustered graphs. Further, these methods are implemented in GEOMI, a visual analysis tool for large and complex networks 1 .

Current work is to further develop the framework for various graph models and application domains.

\footnotetext{
* This research has been partially supported by a grant from the Australian Research Council.
} 


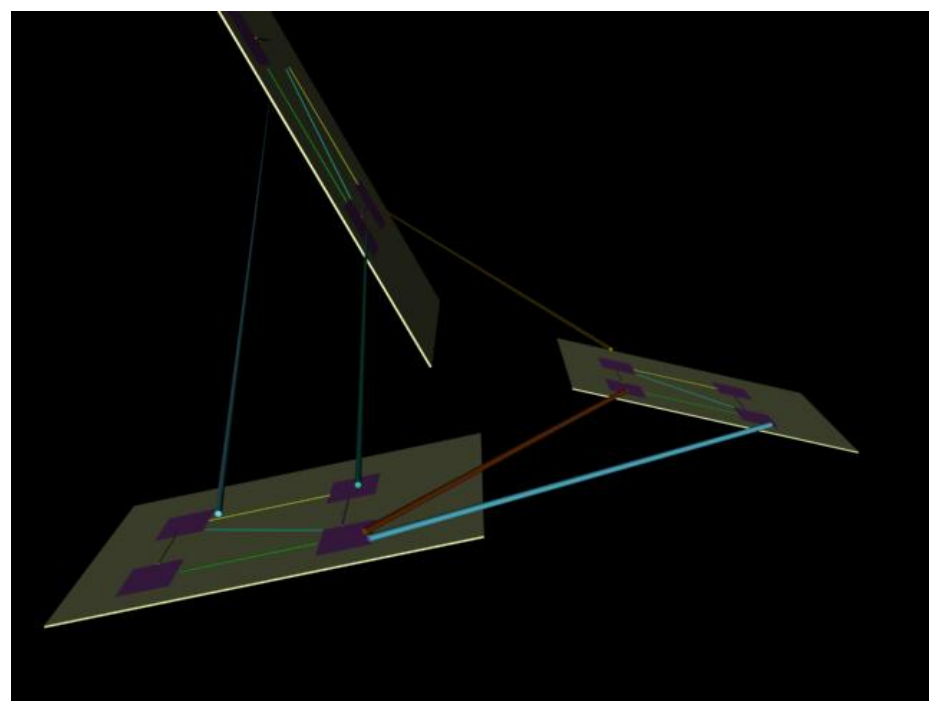

Fig. 1. The MultiPlane framework

\section{References}

1. A. Ahmed, T. Dwyer, M. Forster, X. Fu, J. Ho, S. Hong, D. Koschützki, C. Murray, N. Nikolov, A. Tarassov, R. Taib and K. Xu, GEOMI: GEOmetry for Maximum Insight, Proceedings of Graph Drawing 2005.

2. A. Ahmed, T. Dwyer, S.Hong, C. Murray, L. Song and Y. Wu, Visualisation and Analysis of Large and Complex Scale-free Networks, Proceedings of EuroVis 2005.

3. M. Baur, U. Brandes, M. Gaertler and D. Wagner, Drawing the AS Graph in 2.5 Dimensions, Proceedings of Graph Drawing 2004, Lecture Notes in Computer Science 3383, pp. 43-48, 2004.

4. U. Brandes, T. Dwyer and F. Schreiber, Visualizing Related Metabolic Pathways in Two and a Half Dimensions, Proceedings of Graph Drawing 2003, pp. 111-122, 2003.

5. J. Ho and S. Hong, Drawings Clustered Graphs in Three Dimensions, Proceedings of Graph Drawing 2005.

6. S. Hong and T. Murtagh, Visualization of Large and Complex Networks Using PolyPlane, Proceedings of Graph Drawing 2004, Lecture Notes in Computer Science 3383, pp. 471-482, Springer Verlag, 2004.

7. S. Hong and N. Nikolov, Layered Drawings of Directed Graphs in Three Dimensions, Proceedings of APVIS 2005: Asia Pacific Symposium on Information Visualisation, 2005, CPRIT 45, pp. 69-74, 2005.

8. S. Hong and N. Nikolov, Hierarchical Layouts of Directed Graphs in Three Dimensions, Proceedings of Graph Drawing 2005.

9. C. Ware, Designing with a 2 1/2D Attitude, Information Design Journal 10, 3, pp. 171-182, 2001. 